\title{
Altered posterior cingulate brain metabolites and cognitive dysfunction in preterm adolescents
}

\author{
Jeanie L.Y. Cheong ${ }^{1-3}$, Alan Bainbridge ${ }^{4}$, Peter J. Anderson ${ }^{2,5,6}$, Katherine J. Lee ${ }^{2,6}$, Alice C. Burnett ${ }^{2}$, \\ Deanne K. Thompson ${ }^{2,6-8}$, Gehan Roberts ${ }^{2,69}$, Stephen J. Wood ${ }^{10,11}$, Lex W. Doyle ${ }^{1-3,6}$, Nicola J. Robertson ${ }^{12}$; \\ for the Victorian Infant Collaborative Study Group
}

BACKGROUND: Extremely preterm (EP, <28wk gestation) individuals have increased the risk of cognitive deficits compared with controls. The posterior cingulate region has an important role in cognitive function, but how this is affected by preterm birth is unknown. We aimed to compare brain metabolite ratios of neurons and cell membranes between EP 18-y olds and controls, and explore the association between metabolite ratios and cognitive outcomes.

METHOD: A regional cohort of $150 \mathrm{EP}$ and 134 controls were recruited for the study. Cerebral metabolites were measured using proton magnetic resonance spectroscopy (MRS) obtained from a left posterior cingulate voxel. Total $\mathrm{N}$-acetylaspartate (tNAA, neuronal marker)/total creatine ( $\mathrm{tCr}$ ), and total choline (tCho, cell membrane marker)/tCr ratios were compared between groups using linear regression. Metabolite ratios were correlated with tests of general intelligence $(I Q)$, memory, and attention using linear or logistic regression.

RESULTS: Compared with controls, EP had lower tNAA/tCr (mean difference $(95 \% \mathrm{Cl})$ of $-2.27 \%(-4.09,-0.45))$ and tCho/ $\mathrm{tCr}$ (mean difference $(95 \% \mathrm{Cl})$ of $-11.11 \%(-20.37,-1.85))$, all $P=0.02$. Higher $\mathrm{tCho} / \mathrm{tCr}$ correlated with better IQ in the EP group only; whereas higher tNAA/tCr ratios correlated with better scores in working memory and attention in both groups. CONCLUSION: EP birth is associated with long-term brain metabolite ratio alterations. This may underlie poorer cognitive performance in EP survivors.

A lthough survival rates following extremely preterm (EP, $<28$ wk gestation) birth have increased in the last few decades, significant neurodevelopmental sequelae affects around $25-35 \%$ of survivors (1). These deficits encompass poorer general intelligence (IQ), academic underachievement, behavioral, attentional, socialization problems, and major motor deficits (1-3).

Magnetic resonance imaging (MRI) studies have contributed substantially to the understanding and characterization of preterm brain injury. Brain alterations in preterm children noted on structural $(4,5)$ and diffusion tensor MRI (6) have been shown to correlate with cognitive, motor, and behavioral outcomes in childhood and adolescence $(4,5)$. Brain metabolites measured using magnetic resonance spectroscopy (MRS) may provide insights into the structural changes observed in volumetric and diffusion MRI studies. Using proton $\left({ }^{1} \mathrm{H}\right)$ MRS, metabolites including $\mathrm{N}$-acetylaspartate (NAA), choline-containing compounds (Cho), and creatine (Cr) can be quantified. NAA is primarily found in neurons and is a marker of neuronal integrity (7). The Cho peak on MRS has a contribution from several choline-containing compounds. High Cho levels are reported in pathologies involving increased cellular density and proliferation of membrane phospholipids (8). The Cr resonance comprises creatine and phosphocreatine, compounds involved in cellular energy metabolism (9). Cr levels remain relatively stable after the first year following birth (10) and are often used as a reference in MRS ratios. Although MRS metabolites are presented either as ratios or absolute concentrations, it is not always feasible to obtain the latter in clinical studies due to time constraints related to the imaging protocol. Most brain MRS studies in preterm populations have been performed during the neonatal period ( $34 \mathrm{wk}$ postmenstrual age up to term-equivalent age) (11-13). There is limited information on MRS brain metabolites and associated functional outcomes in preterm young adults $(14,15)$.

The posterior cingulate cortex is a highly connected brain region with diverse functions that encompass IQ, working memory, attention, and executive function (16), all of which are domains that are impaired in the preterm population $(3,17,18)$. Abnormalities of posterior cingulate brain structure, metabolic activity, and function have been documented in neurodegenerative disorders, healthy ageing, major psychiatric disorders, and traumatic brain injury (16). However, how posterior cingulate metabolites are affected following EP birth is not known.

\footnotetext{
${ }^{1}$ Neonatal Services, Royal Women's Hospital, Melbourne, Australia; ${ }^{2}$ Victorian Infant Brain Studies, Murdoch Childrens Research Institute, Melbourne, Australia; ${ }^{3}$ Department of Obstetrics \& Gynaecology, University of Melbourne, Melbourne, Australia; ${ }^{4}$ University College London Hospitals NHS trust, London, UK; ${ }^{5}$ School of Psychological Sciences, University of Melbourne, Melbourne, Australia; ${ }^{6}$ Department of Paediatrics, University of Melbourne, Melbourne, Australia; ${ }^{7}$ Developmental Imaging, Murdoch Childrens Research Institute, Melbourne, Australia; ${ }^{8}$ Florey Institute of Neurosciences and Mental Health, Melbourne, Australia; ${ }^{9}$ Royal Children's Hospital, Melbourne, Australia; ${ }^{10}$ School of Psychology, University of Birmingham, Edgbaston, UK; ${ }^{11}$ Melbourne Neuropsychiatry Centre, Department of Psychiatry, University of Melbourne and Melbourne Health, Melbourne, Australia; ${ }^{12}$ Institute for Women's Health, University College London, London, UK. Correspondence: Jeanie L.Y. Cheong (Jeanie.cheong@thewomens.org.au) 
To address these research gaps, we aimed to understand the impact of EP birth on posterior cingulate brain metabolites in adolescence, and how the metabolites relate to function. We aimed to (i) compare MRS metabolite ratios that reflect neuronal integrity and cell membranes, i.e., total (t)NAA/tCr and $\mathrm{tCho/tCr}$ between EP and controls, and (ii) explore the relationship between MRS metabolite ratios and IQ, working memory and attention at age $18 \mathrm{y}$ and whether these relationships differ between EP and controls. Given that we have previously reported an association between larger brain white matter volumes and higher IQ (5), we also wanted to explore whether the associations between MRS metabolite ratios and cognitive outcomes were independent of white matter volume in this region. We hypothesized that the EP group would have lower tNAA/tCR and tCho/tCr compared with term controls, and that higher $\mathrm{tNAA} / \mathrm{tCr}$ within the posterior cingulate would predict better IQ, working memory and attention, independent of white matter volume.

\section{RESULTS}

For the 18-y follow-up study, 172 (76\% of those alive at 8 y of age) of the EP group and 160 (63\%) of the term-control group

Table 1. Participant characteristics

\begin{tabular}{|c|c|c|}
\hline & $\begin{array}{l}\text { Extremely preterm } \\
\qquad(n=150)\end{array}$ & $\begin{array}{l}\text { Term control } \\
(n=134)\end{array}$ \\
\hline Maternal education $<12 y$ & $74(51), n=145$ & $42(32.3), n=130$ \\
\hline Lower social class ${ }^{\mathrm{a}}$ & $47(32.4), n=145$ & $25(19.1), n=131$ \\
\hline Multiple birth & $52(34.7)$ & $0(0)$ \\
\hline $\begin{array}{l}\text { Gestational age at birth—weeks, } \\
\text { mean (SD) }\end{array}$ & $25.8(1)$ & $39.3(1.3)$ \\
\hline Birthweight—grams, mean (SD) & $895(176)$ & $3440(456)$ \\
\hline $\begin{array}{l}\text { Small for gestational age } \\
\text { (Birthweight Z-score }<-2 \text { SD) }\end{array}$ & $4(2.7)$ & $0(0)$ \\
\hline Male & $72(48)$ & $57(42.5)$ \\
\hline Antenatal corticosteroids & $103(68.7)$ & $0(0), n=133$ \\
\hline $\begin{array}{l}\text { Cystic periventricular } \\
\text { leukomalacia }\end{array}$ & $5(3.3)$ & $0(0)$ \\
\hline $\begin{array}{l}\text { Grade } 3 \text { or } 4 \text { intraventricular } \\
\text { hemorrhage }\end{array}$ & $11(7.3)$ & $0(0)$ \\
\hline Bronchopulmonary dysplasia ${ }^{b}$ & $59(39.3)$ & $0(0), n=133$ \\
\hline Postnatal corticosteroids & $56(37.3)$ & $0(0)$ \\
\hline Cerebral palsy (confirmed at 8 y) & $11(7.5), n=147$ & $0(0), n=132$ \\
\hline
\end{tabular}

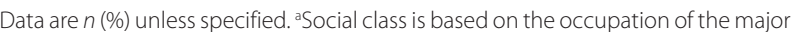
income earner in the family and was categorized as lower (unskilled or unemployed) or higher (semi-skilled, skilled, or professional). 'b Defined as ongoing oxygen requirement at 36 wk corrected age. were seen, of whom $150 \mathrm{EP}$ and 134 controls had MRS data. The mean Cramer-Rao low bounds (expressed as a percentage of the fitted metabolite amplitude) for the included spectra were: tCho $7.8 \pm 3.1 \%$; tNaa $3.0 \pm 0.9 \%$; $\mathrm{tCr} 4.0 \pm 1.0 \%$.

Compared with EP survivors in the original cohort, EP participants who contributed MRS data to this study had higher IQ at 8 y (mean difference $(95 \% \mathrm{CI})$ of $7.0(1.9,12.1))$, lower rates of cerebral palsy at 8 y ( 7.5 vs. $25 \%)$ and less cystic periventricular leukomalacia (3.3 vs. $14.7 \%$ ). Controls with MRS data had higher IQ at $8 \mathrm{y}$ (mean difference $(95 \% \mathrm{CI}$ ) of 6.2 (2.4, $10.1)$ ), were more likely to be singletons (100 vs. $95 \%$ ), and were less likely to have mothers with fewer than $12 \mathrm{y}$ of education (32.3 vs. $46.4 \%$ ) than controls without MRS data. All other characteristics were similar.

Participant characteristics of those included in the current study are summarized in Table 1. Expected differences between groups were seen in all characteristics with the exception of male sex and small for gestational age at birth.

\section{MRS Metabolite Peak-Area Ratios}

Compared with controls, EP adolescents had lower tNAA/tCr and $\mathrm{tCho} / \mathrm{tCr}$ ratios (Table 2). The magnitude of reduction in $\mathrm{tCho} / \mathrm{tCr}$ was almost fivefold that of $\mathrm{tNAA} / \mathrm{tCr}$.

\section{Relationship Between MRS Metabolite Peak-Area Ratios and Outcomes}

IQ, working memory and attention outcomes are summarized in Table 3. Compared with term-born controls, the EP group had lower IQ and performed more poorly in most tasks of working memory and attention, with the exception of immediate memory and selective visual attention.

Metabolite ratios involving tCho were associated with IQ, whereas ratios involving tNAA were associated with working memory and shifting attention (Table 4). Increased tCho/tCr ratios were associated with better IQ; a relationship seen in the EP group only but not controls ( $P$ value for interaction 0.04 ). tNAA/tCr ratios correlated positively with tests of working memory and shifting attention; a relationship that was similar in both EP and control groups ( $P$ value $>0.05$ for all interactions). All relationships between MRS metabolite ratios and IQ, memory, and attention persisted even after adjustment for posterior cingulate white matter volume.

\section{DISCUSSION}

This study demonstrated that brain metabolite ratios $\mathrm{tNAA} / \mathrm{tCr}$ and $\mathrm{tCho} / \mathrm{tCr}$ in the posterior cingulate were lower in $\mathrm{EP}$ survivors in late adolescence compared with controls. These brain metabolite ratios were associated with IQ, working memory,

Table 2. Magnetic resonance spectroscopy metabolite ratios contrasted between the birth groups

\begin{tabular}{|c|c|c|c|c|c|}
\hline Metabolite ratio & $\begin{array}{c}\text { Extremely } \\
\text { preterm }(n=150)\end{array}$ & $\begin{array}{l}\text { Term control } \\
\quad(n=134)\end{array}$ & \multicolumn{2}{|c|}{ Mean difference $(95 \% \mathrm{Cl})$} & $P$ value \\
\hline $\mathrm{tNAA} / \mathrm{tCr}$ & $2.15(0.18)$ & $2.20(0.16)$ & $-0.05(-0.09,-0.01)$ & $-2.27(-4.09,-0.45)$ & 0.02 \\
\hline
\end{tabular}

a Metabolite ratio difference expressed as a percentage of the mean ratio in the controls for the particular metabolite ratio. 


\section{Articles | Cheonget al.}

Table 3. General intelligence, memory, and attention outcomes contrasted between birth groups

\begin{tabular}{|c|c|c|c|c|}
\hline Test variables & Extremely preterm, mean (SD) & Term control, mean (SD) & Mean difference $(95 \% \mathrm{Cl})$ & $P$ value \\
\hline \multicolumn{5}{|l|}{ General intelligence } \\
\hline IQ & $95.9(16.0), n=150$ & $107.0(13.2), n=133$ & $-11.0(-14.5,-7.6)$ & $<0.0001$ \\
\hline \multicolumn{5}{|l|}{ Memory } \\
\hline Immediate memory & $5.7(1.2), n=146$ & $5.9(1.2), n=132$ & $-0.2(-0.5,0.1)$ & 0.25 \\
\hline Working memory & $3.8(1.1), n=146$ & $4.3(1.0), n=132$ & $-0.5(-0.7,-0.2)$ & 0.0002 \\
\hline \multicolumn{5}{|l|}{ Attention } \\
\hline Visual selective attention & $18.2(2.3), n=132$ & $18.7(2.0), n=128$ & $-0.4(-1.0,0.1)$ & 0.10 \\
\hline $\begin{array}{l}\text { Auditory selective attention; median } \\
\text { (interquartile range) }\end{array}$ & $8(5-10), n=130$ & $9(7-10), n=127$ & - & $0.001^{\mathrm{a}}$ \\
\hline $\begin{array}{l}\text { Shifting attention/flexibility; median } \\
\text { (interquartile range) }\end{array}$ & $4(2-7), n=130$ & $7(4-9), n=125$ & - & $<0.001^{\mathrm{a}}$ \\
\hline
\end{tabular}

Table 4. Relationships between magnetic resonance spectroscopy metabolite ratios and cognition, working memory, and attention outcomes across all participants

\begin{tabular}{|c|c|c|c|c|c|c|c|c|}
\hline \multirow[b]{2}{*}{ Outcome } & \multicolumn{4}{|c|}{$\mathrm{tNAA} / \mathrm{tCr}$} & \multicolumn{4}{|c|}{$\mathrm{tCho} / \mathrm{tCr}$} \\
\hline & $\begin{array}{l}\text { Coefficient } \\
(95 \% \mathrm{Cl})^{\mathrm{a}}\end{array}$ & $\begin{array}{c}P \\
\text { value }\end{array}$ & $\begin{array}{c}\text { Adjusted coefficient } \\
(95 \% \mathrm{Cl})^{\mathrm{b}}\end{array}$ & $\begin{array}{l}\text { Adjusted } \\
\text { value }^{\mathrm{b}}\end{array}$ & $\begin{array}{l}\text { Coefficient } \\
(95 \% \mathrm{Cl})^{\mathrm{a}}\end{array}$ & $\begin{array}{c}P \\
\text { value }\end{array}$ & $\begin{array}{c}\text { Adjusted coefficient } \\
\qquad(95 \% \mathrm{Cl})^{\mathrm{b}}\end{array}$ & $\begin{array}{l}\text { Adjusted } \\
P \text { value }^{b}\end{array}$ \\
\hline IQ_EP participants ${ }^{c}$ & $2.0(-10.5,14.5)$ & 0.76 & $-0.5(-13.5,12.6)$ & 0.94 & $12.8(2.8,22.8)$ & 0.01 & $13.5(4.0,23.0)$ & 0.005 \\
\hline $\mathrm{IQ}$-term controls $\mathrm{s}^{\mathrm{C}}$ & $8.5(-5.2,22.3)$ & 0.22 & $9.6(-2.7,21.9)$ & 0.13 & $-4.6(-16.2,6.9)$ & 0.43 & $-1.0(-11.7,9.7)$ & 0.85 \\
\hline Working memory & $0.8(0.1,1.5)$ & 0.02 & $0.7(0.1,1.4)$ & 0.04 & $0.2(-0.4,0.7)$ & 0.56 & $0.2(-0.4,0.7)$ & 0.57 \\
\hline \multicolumn{9}{|l|}{ Attention } \\
\hline Visual selective attention & $0.2(-1.6,1.9)$ & 0.85 & $-0.2(-2.0,1.6)$ & 0.83 & $-0.2(-1.3,1.0)$ & 0.79 & $-0.3(-1.5,0.9)$ & 0.66 \\
\hline Auditory selective attention ${ }^{d}$ & $1.9(0.4,8.4)$ & 0.39 & $1.3(0.3,6.2)$ & 0.73 & $1.1(0.3,3.3)$ & 0.91 & $1.1(0.3,3.4)$ & 0.90 \\
\hline
\end{tabular}

In most analyses, the numbers were slightly lower than this due to missing outcome data (see Table 3 ).

aResults represent the difference in outcome per unit change in the predictor estimated from separate linear regression models for each outcome unless otherwise indicated. ${ }^{\mathrm{b}} \mathrm{Adjusted}$ for posterior cingulate white matter volume. 'Results presented separately for EP and control groups ( $P$ value for interaction 0.04 for $\mathrm{Cho} / \mathrm{Cr}$ ). ${ }^{\mathrm{d}}$ Results from presented as odds ratios from separate logistic regression models for each outcome, where the outcome was scoring $\langle/\rangle$ the median value for the controls.

Measures used: immediate memory (digit recall span), working memory (backward digit recall span), visual selective attention (telephone search task), auditory selective attention (elevator with distraction task), shifting attention/flexibility (elevator with reversal task).

and attention, independent of white matter volume in a similar brain region. Interestingly the relationship between tCho/ tCr with IQ was seen in the EP group but not controls. This requires more study but may suggest that Cho is an important biomarker for cognitive dysfunction in EP survivors.

Unfortunately, we report values from metabolite ratios rather than absolute quantitation. MRS was obtained as part of an extensive MRI protocol and time limitations precluded longer MRS acquisition, which was necessary to obtain absolute metabolite quantitation. Therefore, the lower $\mathrm{tNAA} / \mathrm{tCr}$ ratio in the EP survivors could be due to decreased NAA, increased $\mathrm{Cr}$ or both. NAA is found predominantly in neurons, where concentrations increase in the early stages of gray matter, thalamic, and cerebellar development. This is thought to reflect the rapid formation of dendritic arborizations and synaptic connections (10). In addition, NAA has a role in oligodendrocyte myelin formation, neuroimmune reactions, and intercellular signaling (19). NAA rapidly increases in the first few years following birth, then slows down to reach adult levels by mid-adolescence $(10,20)$. Decreased NAA levels are seen in neurodegenerative disorders, such as Alzheimer's disease and mild cognitive impairment (21), cerebral atrophy in demyelinating and gray matter disorders (22), as well as disorders characterized by a lack of progressive cortical development, such as early onset schizophrenia (23). The impact of preterm birth on NAA levels in adolescence is inconclusive. One study reported lower NAA levels in the hippocampus of preterm 15-y olds compared with controls (14) but another reported similar NAA/Cr ratios in frontal white matter (15). Lower NAA levels in EP compared with controls in this study 
may reflect impairment in the early developmental formation of dendritic arborizations and synaptic connections, which persist into adolescence $(10,23)$.

Developmentally, Cho concentrations in the brain decrease in the first year after birth and remain relatively constant until early adulthood (10). Lower tCho/tCr ratios in the EP group could be a result of decreased Cho, increased $\mathrm{Cr}$, or both. The lower tCho/tCr ratios in the EP group suggest decreased membrane phospholipid content in the posterior cingulate. This may be a result of disrupted Cho metabolism of membrane phospholipids following preterm birth, reflecting altered phospholipid turnover and development with potentially reduced content of dendrites and synapses, as well as cholinergic neurons (24). Our findings do not concur with a previous report where $\mathrm{Cho} / \mathrm{Cr}$ ratios in the frontal white matter were similar between preterm and control adolescents (15). However, in that study, the sample size was small (18 term controls and 18 very-low-birth-weight adolescents) and the MRS was obtained from a different region of the brain. There is no current data on MRS changes in the posterior cingulate region following EP birth. In the current study, we regarded $\mathrm{Cr}$ as a reference for NAA and Cho. Cr levels remain relatively stable after the first year following birth (10), however, caution is needed as $\mathrm{Cr}$ levels can change acutely in pathological conditions in experimental models (25).

The relationship between brain metabolite ratios in infancy and later developmental outcomes in preterm infants has been reported in early childhood, with little information in late adolescence. NAA/Cho ratios in the cerebellum positively correlated with cognitive scores on the Bayley-III developmental assessment at 2 y (13), whereas higher NAA/Cho but lower $\mathrm{Cho} / \mathrm{Cr}$ ratios in the posterior periventricular white matter were associated with better motor development at 1 y (11). Another study, however, did not find an association between NAA/Cho ratios in the deep nuclear gray matter and general development at 18 mo to $2 \mathrm{y}$ (26). In the current study, higher $\mathrm{tCho} / \mathrm{tCr}$ ratios in adolescence were associated with better IQ scores, whereas higher $\mathrm{tNAA} / \mathrm{tCr}$ ratios were associated with better working memory and attention scores in adolescence. The $\mathrm{tCho/tCr}$-function relationships in adolescence are not reflective of the associations described by others in infancy. This may be a result of different underlying processes affecting brain metabolite-function many years after preterm birth.

There are, however, data from other adult-onset conditions that support the association between brain NAA and Cho and cognitive function. Higher NAA/Cr and lower $\mathrm{Cho} / \mathrm{Cr}$ are associated with cognitive functioning in Alzheimer's disease, mild cognitive impairment, schizophrenia, and traumatic brain injury $(21,27)$. In Alzheimer's disease and mild cognitive impairment, NAA/Cr ratios in the posterior cingulate region decreased with cognitive and memory decline associated with disease progression $(21,27)$. This is in contrast with the normal ageing process where increases in NAA, possibly neuronal hypertrophy, and Cho, reflecting glial proliferation, have been documented (28). In our study, we found that the relationships between metabolites and IQ were mostly involving

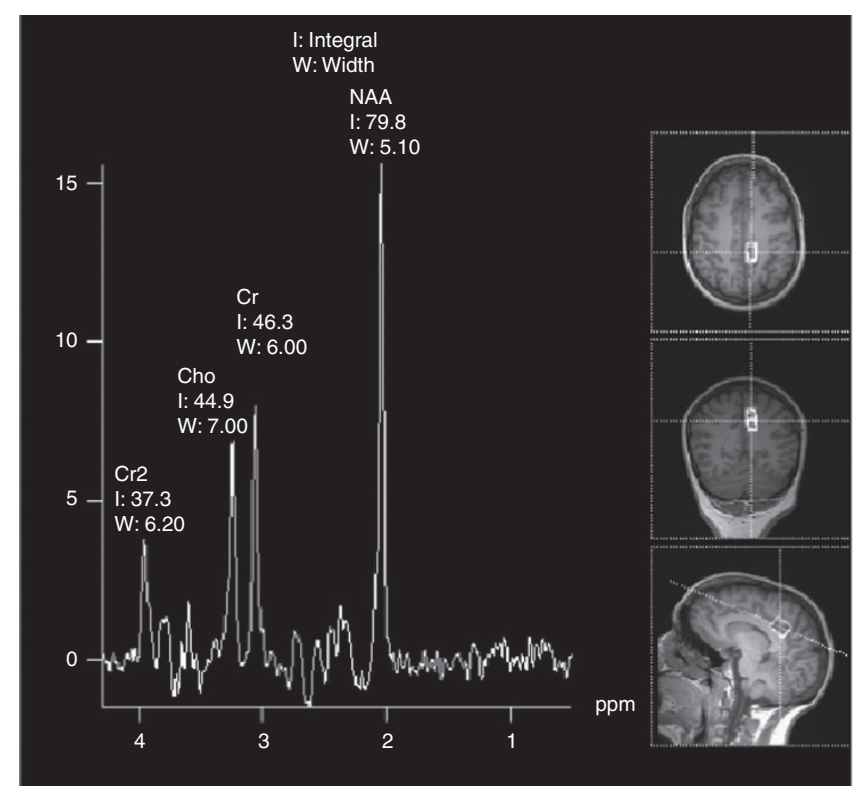

Figure 1. Representative proton spectra and voxel placement centred on the left posterior cingulate region.

Cho, whereas those with working memory and attention were related to NAA. Of interest was the differential relationship between Cho ratios and IQ for the EP and control groups. This may suggest vulnerability of choline-containing compounds following EP birth, and may reflect either delayed development or early decline of Cho. Further research into potential alterations of choline function in preterm survivors is needed, given the paucity of such studies in the current literature.

We have previously reported in this same cohort that volumes of multiple brain tissues and structures were smaller in the EP group compared with controls (5). Of particular relevance to this study was white matter volume; which was in the order of $6.0 \%$ smaller ( $95 \%$ confidence interval of $-8.8 \%$ to $-3.2 \%)$ in the EP group compared with controls. In the present study, brain metabolites sampled from a specified region of white matter (left posterior cingulate) had lower tNAA/ $\mathrm{tCr}$ and $\mathrm{tCho} / \mathrm{tCr}$ in EP compared with controls. Thus, we can speculate that not only is EP birth associated with long term effects on brain volumes, it is also associated with lower concentrations of brain metabolites that are markers of neurons and cell membranes within the white matter. In addition, the previous study also reported an association between larger brain white matter volumes and higher IQ scores. An interesting finding from the current study involves the relationships between MRS metabolite ratios and cognitive outcomes (IQ, memory, and attention) which are independent of posterior cingulate white matter volumes. This may suggest that following EP birth, brain metabolite ratios are influencing cognitive function via alternative pathways to brain volumes, and highlights the need for further research into understanding these pathways.

This study is the largest neuroimaging cohort of EP and term-born controls in the era of modern intensive care to be prospectively followed up to late adolescence. With a 
contemporaneous group of term-born controls, we were able to make direct group comparisons of our MRS data rather than relying on normative data, which may not accurately reflect what is seen in our population. In addition, there were functional outcome data from validated neuropsychological tests that investigated functions associated with the posterior cingulate cortex. Thus, we were able to further understand how these metabolites are related to cognitive functioning in EP adolescents. The differential relationship between tCho/ tCr and IQ between EP and term controls seen in our study is a novel finding. This opens up the possibility of further research into how Cho may be differentially affected following preterm birth.

Although a high proportion of participants consented to have MRI and MRS examinations, outcomes were only available for $60-78 \%$ of those originally recruited. EP participants who returned for assessment had higher IQ at age $8 \mathrm{y}$, lower rates of brain injury and were less likely to have cerebral palsy compared with EP participants who were not assessed. Similarly, control participants who were assessed had higher IQ at $8 \mathrm{y}$ and additionally were more likely to have mothers with greater than $12 \mathrm{y}$ of education, than controls who did not return for assessment. Thus, the differences we have observed may have been greater if the whole cohort had been assessed. Finally, we had cross sectional MRS data rather than longitudinal data, which limited our ability to understand the trajectory of brain metabolite differences between the birth groups from birth until adolescence.

We identified brain metabolite profiles that reflect altered development involving dendrites, synaptic formation and membranes in EP infants in late adolescence. This may underlie the association of EP birth with poorer performance on cognitive tasks. Of concern was little evidence of developmental "catch-up" of brain metabolites in late adolescence. It thus highlights the importance of understanding how EP birth potentially impacts on the normal ageing process as more EP survivors reach adulthood.

\section{METHODS}

\section{Participants}

Participants were part of a prospective longitudinal cohort study of all EP adolescents born in the state of Victoria, Australia between 1991 and 1992. Term-born controls were contemporaneously recruited at birth, matched to the EP cohort on expected date of birth, mother's country of birth (English-speaking versus other) and health insurance status (private or public). Follow up at ages 2 (29), 5 (30), and 8 y have been previously reported (31). At age $18 \mathrm{y}$, participants attended a comprehensive health and developmental assessment including MRI and MRS. Written informed consent was obtained from all participants, including their parents if aged less than $18 \mathrm{y}$ at the time of assessment. Ethical approval for the original and follow-up studies were obtained from the Human Research and Ethics committees of the four participating centers, i.e., the Royal Women's Hospital, Mercy Hospital for Women, Monash Medical Centre and Royal Children's Hospital, Melbourne.

The MRI volumetric data, IQ, and the attention data have previously been reported on this cohort $(5,32,33)$. In this manuscript, we report the MRS data and how that relates to IQ, working memory, and attention.

\section{MRS}

MRS was performed at two study sites, each using a Siemens 3T MAGNETOM Trio MRI system (Siemens Healthcare, Erlangen,
Germany), a 12-channel receive-only head coil, and the same acquisition protocol. Proton $\left({ }^{1} \mathrm{H}\right)$ MRS was obtained from a $20 \times 15 \times 10 \mathrm{~mm}$ voxel centred on the left posterior cingulate white matter (Figure 1) using a Point Resolved Spectroscopy (PRESS) acquisition (repetition time $=3,000 \mathrm{~ms}$, echo time $=135 \mathrm{~ms}, 128$ averages). Spectra were quantified using LCModel (34). LCModel fits to in vivo spectra using a linear combination of simulated basis spectra for individual metabolites. The basis set used for this study included NAA, N-acetylaspartate-glutamate (NAAG), choline containing compounds (phosphocholine (PCh) and glycero-phosphocholine (GPC)) and creatine $(\mathrm{Cr})$ and phosphocreatine (PCr). Metabolites were defined as follows: total NAA (tNAA) = NAA+NAAG; total choline $(\mathrm{tCho})=\mathrm{GPC}+\mathrm{PCh}$; total creatine $(\mathrm{tCr})=\mathrm{Cr}+\mathrm{PCr}$. LCModel provides an estimate of the standard deviation (SD) (Cramer-Rao lower bound) of the fitted amplitude of each metabolite included in the basis set expressed as a percentage of the amplitude. Metabolites with SD $>50 \%$ are unreliably determined; major metabolites should have SD $<20 \%$ (35). Each spectrum was manually checked and excluded if all of tNAA, tCho and $\mathrm{tCr}$ had $\mathrm{SD}>20 \%$ indicating a poor quality spectrum. In principle, LCModel allows estimation of absolute concentrations by reference to a separate unsuppressed water acquisition. However, at an echo time of $135 \mathrm{~ms}$, large corrections for both water and metabolite T2 relaxations are required. For this study, there was insufficient time to directly estimate $\mathrm{T} 2$ relaxation and so only the metabolite ratios were calculated.

\section{Outcome Measures}

For the purposes of this study, we focused on tests of functions thought to be associated with the posterior cingulate region, which included IQ, working memory and attention (16). Participants were assessed by trained examiners who were unaware of previous clinical history or group allocation.

IQ

IQ was estimated using a two-subtest version of the Wechsler Abbreviated Scale of Intelligence (WASI) (36). The WASI IQ provides a standardized scale with a mean of 100 and SD of 15.

\section{Working Memory}

The Digit Recall and Backward Digit Recall subtests of the Working Memory Test Battery for Children were administered. In both subtests participants were presented with a sequence of digits of increasing span. In Digit Recall, the participants were required to recall the sequence of digits in the same order as they were presented, while in Backward Digit Recall participants were required to recall the digits in the reverse order. The maximum span, i.e., the longest recall sequence was recorded.

\section{Attention}

Three measures of attention, all derived from the Test of Everyday Attention (37), were used.

(i) Visual selective attention: The Telephone Search task involved identifying target stimuli (maximum $=20$ ) that were interspersed amongst distractor stimuli in a simulated telephone directory.

(ii) Auditory selective attention: The Elevator with Distraction task involved participants imagining that they were in an elevator in which the visual floor indicator was not working. Participants were asked to calculate which floor they were located by counting a series of specific tones while ignoring the presence of a distractor tone. Ten trials were administered and the variable of interest was the number of correct trials (range 0-10).

(iii) Shifting attention: The Elevator with Reversal task required participants to count strings of medium pitched tones. Interspersed with these medium pitched tones were both high (indicating that the subject must switch to counting up) and low tones (indicating that the subject must switch to counting down). Ten trials were administered and the variable of interest was the number of correct trials (range $0-10$ ).

Statistical Analyses

Data were analyzed using STATA 13.0 (StataCorp, College Station, TX). Participant characteristics were compared (i) between EP and 
controls, as well as (ii) participants with and without analyzable MRS within the EP and control groups, using $t$-tests and chi squared. Group differences in MRS metabolite ratios were explored using linear regression fitted using generalized estimating equations with robust (sandwich) estimation of standard errors to allow for multiple births within a family, using separate models for each brain metabolite ratio. IQ, memory, and attention variables were compared between groups using $t$-tests, or Wilcoxon rank sum tests for nonnormal data. Unlike IQ scores, which were age-appropriate, the working memory and attention results were presented as raw scores and thus, analyses were repeated adjusted for corrected age at assessment. The relationships between brain metabolite ratios and functional outcomes (that were approximately normally distributed) were explored using linear regression, again fitted using generalized estimating equations with robust (sandwich) estimation of standard errors, with a separate model for each metabolite ratio-outcome combination. For functional outcome variables that had a skewed distribution, we used logistic regression with the outcomes dichotomized using the median for the particular variable as the cut-point. To determine whether the relationship between the metabolite ratio and outcome was the same in the EP and control groups, we used an interaction effect. To determine whether MRS metabolite ratios independently predicted IQ, memory, and attention, we added left posterior cingulate white matter volume as an additional step to the regression. Left posterior cingulate white matter volume was automatically generated from the $\mathrm{T}_{1}$-weighted structural MRI scan using FreeSurfer software as previously described (5).

We acknowledge the multiple comparisons within this study and hence we interpret our findings by focusing on overall patterns and magnitude of differences, rather than on individual $P$ values.

\section{STATEMENT OF FINANCIAL SUPPORT}

This work was supported by the Australian National Health and Medical Research Council (Project Grant ID 491246 to L.D., P.A., S.W., J.C., as well as C Robertson, S Hope, and D Hacking; Centre of Clinical Research Excellence Grant ID 546519; Senior Research Fellowship ID 628371 to P.A.; Early Career Fellowship ID 1053787 to J.C.; Early Career Fellowship ID 1012236 to D.T.; Career Development Fellowship ID 1053609 to K.J.L.), the UK Department of Health's NIHR Biomedical Research Centres funding scheme to $A B$, and the Victorian Government Operational Infrastructure Support Program. The authors declare no conflict of interest.

Disclosure: None.

\section{VICTORIAN INFANT COLLABORATIVE STUDY GROUP MEMBERS}

Convenor; Lex W Doyle, MD, FRACP1,23,5; Collaborators (in alphabetical order): Peter J Anderson, $\mathrm{PhD}^{3,5}$, Alice Burnett, $\mathrm{PhD}^{1,5}$, Catherine Callanan, $\mathrm{RN}^{1}$, Elizabeth Carse, FRACP${ }^{7}$, Margaret $\mathrm{P}$ Charlton, $\mathrm{M}$ Ed Psych ${ }^{7}$, Jeanie Cheong, MD, FRACP1,2,5, Cinzia R De Luca, BSc, PhDD ${ }^{1,2}$, Marie Hayes, RN ${ }^{7}$, Esther Hutchinson, BSc (Hons) ${ }^{1}$, Elaine Kelly, $\mathrm{MA}^{16}$, Marion McDonald, RN ${ }^{1}$, Carly Molloy, PhD ${ }^{1,2}$, Gillian Opie, FRACP 6 , Gehan Roberts, MPH, PhD, FRACP1,3,5, Colin Robertson, MD, FRACP8, Karissa Searle', Andrew Watkins, FRACP6, Amanda Williamson ${ }^{6}$, Michelle Wilson-Ching, PhD ${ }^{1,2}$, Stephen J Wood, PhD ${ }^{4,9}$, Heather Woods, $\mathrm{RN}^{6}$. From: the ${ }^{1}$ Premature Infant Follow-up Program at the Royal Women's Hospital, the Departments of 'Obstetrics and Gynaecology, ${ }^{3}$ Paediatrics and ${ }^{4}$ Psychiatry at The University of Melbourne, ${ }^{5}$ Murdoch Childrens Research Institute, ${ }^{6}$ Mercy Hospital for Women, ${ }^{7}$ Monash Medical Centre, and the ${ }^{8}$ Royal Children's Hospital, Melbourne, Australia, and ${ }^{9} S c h o o l$ of Psychology, the University of Birmingham, United Kingdom.

\section{REFERENCES}

1. Saigal S, Doyle LW. An overview of mortality and sequelae of preterm birth from infancy to adulthood. Lancet 2008;371:261-9.

2. Serenius F, Källén K, Blennow M, et al.; EXPRESS Group. Neurodevelopmental outcome in extremely preterm infants at 2.5 years after active perinatal care in Sweden. JAMA 2013;309:1810-20.

3. Hutchinson EA, De Luca CR, Doyle LW, Roberts G, Anderson PJ; Victorian Infant Collaborative Study Group. School-age outcomes of extremely preterm or extremely low birth weight children. Pediatrics 2013;131: e1053-61.
4. de Kieviet JF, Zoetebier L, van Elburg RM, Vermeulen RJ, Oosterlaan J. Brain development of very preterm and very low-birthweight children in childhood and adolescence: a meta-analysis. Dev Med Child Neurol 2012;54:313-23.

5. Cheong JL, Anderson PJ, Roberts G, et al. Contribution of brain size to IQ and educational underperformance in extremely preterm adolescents. PLoS One 2013;8:e77475.

6. Mukherjee P, Miller JH, Shimony JS, et al. Diffusion-tensor MR imaging of gray and white matter development during normal human brain maturation. AJNR Am J Neuroradiol 2002;23:1445-56.

7. Birken DL, Oldendorf WH. N-acetyl-L-aspartic acid: a literature review of a compound prominent in $1 \mathrm{H}-\mathrm{NMR}$ spectroscopic studies of brain. Neurosci Biobehav Rev 1989;13:23-31.

8. Stork C, Renshaw PF. Mitochondrial dysfunction in bipolar disorder: evidence from magnetic resonance spectroscopy research. Mol Psychiatry 2005;10:900-19.

9. Wyss M, Kaddurah-Daouk R. Creatine and creatinine metabolism. Physiol Rev 2000;80:1107-213.

10. Pouwels PJ, Brockmann K, Kruse B, et al. Regional age dependence of human brain metabolites from infancy to adulthood as detected by quantitative localized proton MRS. Pediatr Res 1999;46:474-85.

11. Kendall GS, Melbourne A, Johnson S, et al. White matter NAA/Cho and $\mathrm{Cho} / \mathrm{Cr}$ ratios at MR spectroscopy are predictive of motor outcome in preterm infants. Radiology 2014;271:230-8.

12. Xu D, Bonifacio SL, Charlton NN, et al. MR spectroscopy of normative premature newborns. J Magn Reson Imaging 2011;33:306-11.

13. Van Kooij BJ, Benders MJ, Anbeek P, Van Haastert IC, De Vries LS, Groenendaal F. Cerebellar volume and proton magnetic resonance spectroscopy at term, and neurodevelopment at 2 years of age in preterm infants. Dev Med Child Neurol 2012;54:260-6.

14. Gimenez M, Soria-Pastor S, Junque C, et al. Proton magnetic resonance spectroscopy reveals medial temporal metabolic abnormalities in adolescents with history of preterm birth. Pediatr Res 2008;64:572-7.

15. Bathen TF, Sjöbakk TE, Skranes J, et al. Cerebral metabolite differences in adolescents with low birth weight: assessment with in vivo proton MR spectroscopy. Pediatr Radiol 2006;36:802-9.

16. Leech R, Sharp DJ. The role of the posterior cingulate cortex in cognition and disease. Brain 2014;137(Pt 1):12-32.

17. Omizzolo C, Scratch SE, Stargatt R, et al. Neonatal brain abnormalities and memory and learning outcomes at 7 years in children born very preterm. Memory 2014;22:605-15.

18. Anderson PJ, De Luca CR, Hutchinson E, Spencer-Smith MM, Roberts G, Doyle LW; Victorian Infant Collaborative Study Group. Attention problems in a representative sample of extremely preterm/extremely low birth weight children. Dev Neuropsychol 2011;36:57-73.

19. Baslow MH. N-acetylaspartate in the vertebrate brain: metabolism and function. Neurochem Res 2003;28:941-53.

20. Kreis R, Ernst T, Ross BD. Development of the human brain: in vivo quantification of metabolite and water content with proton magnetic resonance spectroscopy. Magn Reson Med 1993;30:424-37.

21. Kantarci K. Proton MRS in mild cognitive impairment. J Magn Reson Imaging 2013;37:770-7.

22. van der Knaap MS, van der Grond J, Luyten PR, den Hollander JA, Nauta JJ, Valk J. 1H and 31P magnetic resonance spectroscopy of the brain in degenerative cerebral disorders. Ann Neurol 1992;31:202-11.

23. Stanley JA, Vemulapalli M, Nutche J, et al. Reduced N-acetyl-aspartate levels in schizophrenia patients with a younger onset age: a single-voxel $1 \mathrm{H}$ spectroscopy study. Schizophr Res 2007;93:23-32.

24. Tayebati SK, Amenta F. Choline-containing phospholipids: relevance to brain functional pathways. Clin Chem Lab Med 2013;51:513-21.

25. Kulak A, Duarte JM, Do KQ, Gruetter R. Neurochemical profile of the developing mouse cortex determined by in vivo $1 \mathrm{H}$ NMR spectroscopy at $14.1 \mathrm{~T}$ and the effect of recurrent anaesthesia. J Neurochem 2010;115:1466-77.

26. Augustine EM, Spielman DM, Barnes PD, et al. Can magnetic resonance spectroscopy predict neurodevelopmental outcome in very low birth weight preterm infants? J Perinatol 2008;28:611-8. 


\section{Articles | Cheonget al.}

27. Schott JM, Frost C, MacManus DG, Ibrahim F, Waldman AD, Fox NC. Short echo time proton magnetic resonance spectroscopy in Alzheimer's disease: a longitudinal multiple time point study. Brain 2010;133:3315-22.

28. Chiu PW, Mak HK, Yau KK, Chan Q, Chang RC, Chu LW. Metabolic changes in the anterior and posterior cingulate cortices of the normal aging brain: proton magnetic resonance spectroscopy study at $3 \mathrm{~T}$. Age (Dordr) 2014;36:251-64.

29. The Victorian Infant Collaborative Study Group. Outcome at 2 years of children 23-27 weeks' gestation born in Victoria in 1991-92. J Paediatr Child Health. 1997;33:161-5.

30. Doyle LW; Victorian Infant Collaborative Study Group. Outcome at 5 years of age of children 23 to 27 weeks' gestation: refining the prognosis. Pediatrics 2001;108:134-41.

31. Doyle LW, Anderson PJ; Victorian Infant Collaborative Study Group. Improved neurosensory outcome at 8 years of age of extremely low birthweight children born in Victoria over three distinct eras. Arch Dis Child Fetal Neonatal Ed 2005;90:F484-8.
32. Cheong JL, Burnett AC, Lee KJ, et al. Association between Postnatal Dexamethasone for Treatment of Bronchopulmonary Dysplasia and Brain Volumes at Adolescence in Infants Born Very Preterm. J Pediatr. 2014;164:737-43.e1.

33. Wilson-Ching M, Molloy CS, Anderson VA, et al. Attention difficulties in a contemporary geographic cohort of adolescents born extremely preterm/extremely low birth weight. J Int Neuropsychol Soc 2013;19: 1097-108.

34. Provencher SW. Automatic quantitation of localized in vivo $1 \mathrm{H}$ spectra with LCModel. NMR Biomed 2001;14:260-4.

35. Provencher SW. LCModel User's Manual. Version 6.3. San Diego, CA: LC Model Inc., 2013.

36. Wechsler D. Wechsler Intelligence Scale for Children. 3rd edn. San Antonio, TX: The Psychological Corporation, 1991.

37. Robertson IH, Ward T, Ridgeway V, Nimmo-Smith I. The structure of normal human attention: The Test of Everyday Attention. J Int Neuropsychol Soc 1996;2:525-34. 